\title{
Prevalence of serum antibodies against vaccine-preventable diseases in Japanese students and its advisory standard of vaccination
}

\author{
Kei Numazaki* \\ Division of International Infectious Diseases, Graduate School of Health and Welfare, International University of Health and Welfare, Otawara, Tochigi, 324-8501 Japan
}

\begin{abstract}
Although measles, mumps, rubella, and varicella (MMRV) are vaccine-preventable diseases common in childhood, the incidence of these viral infections has recently increased in the adult Japanese population. For prevention against infections in medical facilities, it is important to assess the immunity against these viruses and to achieve immunocompetence by vaccination. The purpose of this study was to determine the need for MMRV vaccinations in Japanese students of co-medical fields by means of a seroprevalence survey for these viral infectious diseases and to recommend vaccination.

Status of the students attending to International University of health and welfare, Otawara, Tochigi, Japan were surveyed in 2008 to 2011 academic year for vaccination and past medical history by self-administered questionnaire and the serum antibody prevalence for enrollment concerning measles, rubella, varicella and mumps. Majority of the students in all years had immunity for measles, rubella and varicella but not for mumps by EIA assy. Although positive rates of measles antibody had risen since 2009, which of rubella was extremely low in the year of 2011. Serum antibody titers of rubella were lower than those of measles in all years. Freshman of 2008 showed highest antibody titers against measles and rubella. A high prevalence rate for the varicella antibody was observed in subjects of 2010 and 2011. The prevalence rate for the mumps antibody was lower in subjects of 2010 and 2011.

Vaccinations in Japan are not enforced and like the United States for final vaccination activities entrusted to guardians and personal perceptions and judgment, limits in encouraging vaccination for legal status. The results of this study suggest that the forth stage of measles and rubella mixed regular vaccination targeted since 2008 in Japan was effective. Also determined what should be done the encouraging vaccination based on specific past medical history, vaccination histories (including dates and times) about encouraging vaccination, appropriate for the individual.
\end{abstract}

\section{Introduction}

Since vaccines containing measles, rubella, mumps and varicella (MMRV) vaccine viruses were licensed, the numbers of reported cases of measles, mumps, rubella, and chickenpox have dramatically decreased [1]. Since 2012, fewer cases of measles, rubella, and mumps have been reported than at any time since nationwide disease reporting began, and elimination of indigenous transmission of Japan appears feasible.

Measles can be severe and encephalitis occurs in approximately one of every 1,000 reported cases. As death occurs in 1-2 of every 1,000 reported measles cases, the risk for severe measles complications is greater for infants, young children. The most ordinary causes of death are associated with pneumonia and encephalitis. In developing countries, measles is often more severe and the case-fatality rate can be as high as $25 \%$. Subacute sclerosing panencephalitis (SSPE) is a rare complication of the central nervous system associated with measles. Among immunocompromised individuals, measles may happen without the typical skin rash and infected persons may shed measles virus for several weeks after the acute phases.

Rubella is febrile exanthematous illness characterized by mild clinical symptoms including transient erythematous and pruritic rash, postauricular or suboccipital lymphadenopathy, arthralgia, and lowgrade fever. Moreover, some of rubella infections are asymptomatic. The most important consequences of rubella infection during early pregnancy, especially during the first trimester are the miscarriages, stillbirths and fetal anomalies. The anomalies most commonly associated with congenital rubella syndrome (CRS) are sensorineural deafness, cataracts, microphthalmia, glaucoma, chorioretinitis, congenital heat anomalies, diabetes and neurologic disorders. Infants with CRS frequently exhibit both intrauterine and postnatal growth retardation. The primary aim of the rubella immunization is the prevention of CRS.

Asymptomatic mumps infections are more common among adults than children, most serious complications of mumps are orchitis, sterility, aseptic meningitis and sensorineural deafness. As varicella are vaccine-preventable diseases (VPD) are important in the health and welfare of infection control perspective, positive control serum antibody or vaccine and its effects have been reported [1].

Although the hemagglutination-inhibition (HI) test used to be the most commonly used laboratory test for assessing immunity,

${ }^{*}$ Correspondence to: Kei Numazaki, MD, PhD, Professor \& Chair, Division of International Infectious Diseases, Graduate School, International University of Health and Welfare, 2600-1 Kitakanemaru, Otawara, Tochigi, 324-8501 Japan, Tel: +81-287-24-3000; Fax: +81-287-24-3100; E-mail: numazaki@iuhw.ac.jp

Key words: measles, rubella, varicella, mumps, vaccination

Received: February 26, 2021; Accepted: March 11, 2021; Published: March 15, 2021 
more sensitive enzyme immunoassay (EIA) assays are now used in most diagnostic laboratories [2]. All persons who work in health-care facilities should be immune to MMRV and have a responsibility to avoid transmitting infections and thereby causing harm to patients.

In 2009, the Regional Committee of the Western Pacific Region Office (WPRO) of WHO formally declared a regional measles elimination target date of 2012 [3,4]. Reported cases of measles in Japan which was 11,015 in 2008 decreased to 32 in 2016. This fact was estimated due to the effect of third and fourth routine vaccination opportunity for measles and rubella (MR) started in the year of 2006 by the Japanese Ministry of Health and Labor. The two dose of immunization program with MR vaccine has been started since April $2006[5,6]$. But not yet reached the number of patients achieving measles elimination required. The third and fourth routine vaccination rates also have been too low.

In previous study [5] for the establishment of immediate infection control system for future construction, we made analysis of serum prevalence of antibodies of Japanese students in co-medical fields and with a history of disease and vaccination history. The purpose of the study was to determine the need for MMRV vaccinations in students by means of a seroprevalence survey for these viral infectious diseases and to recommend vaccination.

\section{Prevalence of serum antibodies against VPD in Japa- nese students of co-medical fields}

Serum samples for all students in the International University do Health and Welfare (IUHW), Otawara, Tochigi, Japan were examined by the serum antibody titers at the time of admission to the university [5]. One hundred and seventy- one students in the year of 2008, 256 students in 2009, 265 students in 2010 and 300 in 2011 were analyzed about vaccination and past medical history of each disease. Selfadministered questionnaire survey, antibodies and related factors were analyzed. Serum was separated from other blood components and kept refrigerated at $4^{\circ} \mathrm{C}$ until testing. All study procedures were approved by the research ethics committee of IUHW.

Serum antibodies to measles, varicella and mumps were determined by EIA and those to rubella were determined by HI assay [2]. Until the year of 2009 antibodies to varicella and mumps had been determined by complement-fixation (CF) test and by HI assay. EIA index more than 4.0, HI titers more than 1:16 and CF titers more than 1:4 were considered for positive. Recommendation for vaccination was sent in writing to all subjects with negative antibody titers. Statistical data analyses were conducted using SPSS ${ }^{\circledR}$ software. Continuous parameters with normal distribution were analyzed by Student's t-test. Binary variables were analyzed by the chi-square and Fisher exact tests. A twotailed test was used for all statistical analyses. In all cases, $p=0.05$ was considered as the threshold.

Measles antibody-positive rates of 2008-2011 were approximately 94 to $99 \%$. The prevalence rate for measles antibody was higher in all years; however, difference was not statistically significant. Those to rubella were approximately 80 to $94 \%$, to chickenpox were approximately $40-41 \%$ by CF, approximately 92 to $93 \%$ by EIA and to mumps were approximately 11 to $12 \%$ by $\mathrm{HI}$, approximately 72 to $73 \%$ by EIA. Measles and rubella antibody positive rates rose in 2009 and 2010. The prevalence rate for the rubella antibody was significantly lower in subjects of 2011 ( $\mathrm{p}=0.005)$. A high prevalence rate for the varicella antibody by EIA was observed in subjects of 2010 and 2011 . The prevalence rate for the mumps antibody by EIA was lower in subjects of 2010 and 2011. Average antibody titers to measles of
2008-2011 were approximately 22 to 25 EIA index. Those to rubella by $\mathrm{HI}$ assay of 2008, 2009-2011 were 1:50 to $1: 80$. Significantly higher distribution of average measles and rubella antibody titers were found in $2008(\mathrm{p}=0.005)$.

\section{Advisory standard of vaccination for clinical training}

Both serologic and epidemiologic evidence indicate that the vaccine induces lifelong immunity in most persons [6]. Findings of some studies indicate that immunity can wane after first vaccination and this phenomenon appears to have some effects on measles transmission and the occurrence of outbreaks [6-8]. There are few clinically-useful rapid diagnoses for modified measles over the world $[9,10]$. Measles was estimated to infect 20 million people per year worldwide, and to result in 242,000 deaths [1,11].

Although MMRV are common in childhood, the incidence of these viral infections has increased in the adult and young Japanese population [1]. The risk for death from measles or its complications is greater for infants, young children [11]. Serologic screening for MMRV immunity generally is necessary only when persons identified as susceptible are subsequently vaccinated in a timely manner $[11,12]$. Since measles vaccine became available, professional and voluntary medical and public health organizations have collaborated in vaccination programs that have reduced the reported incidence of measles by greater than $99 \%[1,6]$. Even countries that MMRV infections had been eradicated still have the high risk of importation from countries that have not yet eliminated the disease [3]. For prevention against MMRV infection in medical facilities, it is important to assess the immunity against these viruses and to achieve immunocompetence by useful vaccination $[3,13]$.

Although Japanese regular measles and rubella immunization has been changed to two-dose schedule, introducing live attenuated MR combined vaccine since April 2006, the first immunization (1224 months after birth) and the second one (5-6 years, less than 1 year before primary school entrance), there are still estimated 1 to 10 hundred measles cases and several measles deaths annually in Japan. This epidemic of combined MR vaccine was a just when the inoculation was started 2 times next year that had predicted a pandemic outbreak. The decrease in measles cases owes much to the raising up in the measles vaccination rate among 1-2 years children by success of countermeasures taken by the whole country, academic societies, local governments, and medical associations since 2001. Also missing a kit for lack of vaccination or measles antibody measurement and social disruption occurs.

The third and fourth periodic inoculation of MR combined vaccine has been induced since 2008. Be under 18 years old, school-aged population vaccination 2 opportunities for measles and rubella vaccine given supplementary vaccination 2013 onwards. Vaccinations in Japan are not enforced and like the United States for final vaccination activities entrusted to guardians and personal perceptions and judgment, limits in encouraging vaccination for legal status. Environment surrounding the vaccination in Japan in 2012, measles elimination goals are in jeopardy began to move forward at last in recent years an increasing as any approval of a new vaccine.

Most serious complications of mumps are more common among youth and adults than among children [14,15]. Although orchitis may occur among up to $38 \%$ of young adult men in whom mumps develops, sterility is thought to occur not rarely. Aseptic meningitis affects $4 \%$ $6 \%$ of persons with clinical cases of mumps and symptom is usually mild. In the pre-vaccination era, mumps was one of the major causes 
of sensorineural deafness among children. Deafness may be sudden in onset, bilateral, and permanent.

Infection defense can be antibody prevalence of measles, rubella, and varicella is results of previous study [5], population immunity has had inadequate in and about mumps. Low vaccination rates are a chickenpox comes high natural incidence rates are estimated factors. Effect of phase $4 \mathrm{MR}$ vaccination routine suggested as its influence, in MR antibody prevalence rises observed in year of 2009 and 2010. Due to changes in inspection methods considered difference between antibody prevalence of varicella, mumps grade, determined to assay system for fiscal 2010 better suited antibody screening in terms of sensitivity, specificity. However, the detection level of antibodies to defend is not clearly EIA laws but not necessarily.

Therefore, considered thorough inoculation with immune acquisition rate is higher as 2 doses are effective in infection prevention practices. But for integrated University's standards are not necessarily equipped with advanced data analysis also untested on infection prevention measures inadequate conditions during clinical practice [16]. A confirmation of antibody acquisition and inoculation encouragement manages the accurate information about the history of the student unless the possibility of an appropriate individual [5]. Future vaccination rates improved and common understanding for more students to investigate the trends of the University as a whole, public should have the flexibility.

The criteria for acceptable evidence of immunity to MMR provide presumptive rather than absolute evidence of immunity [17]. In the past, the most commonly used laboratory test for assessing immunity to measles was the HI test but more sensitive assays such as EIA are now used in most laboratories. Serologic screening for MMR immunity generally is not necessary nor if a person has other acceptable evidence of naturally acquired immunity to the disease. Serologic screening is appropriate only when persons identified as susceptible are subsequently vaccinated in a timely manner. Screening is most applicable when the return and vaccination of those tested can be ensured $[17,18]$. If these conditions are not met, serologic screening is inappropriate. Likewise, during an outbreak of MMRV, serologic screening before vaccination generally is not recommended because the rapid vaccination needed to curb the outbreak.

\section{Conclusions}

Colleges, universities, technical and vocational schools, and other institutions for post-high school education should require that all undergraduate and graduate students have received two doses of MMRV vaccine or have other acceptable evidence of measles, rubella, and mumps immunity before enrollment. Suggested vaccine immunocompromised persons regardless of the number of inoculations in the meantime, cannot get the antibody presence suggests the antibodies after vaccination review during clinical practice and matter management. To encouraging vaccination shall be is determined to what should be done the encouraging vaccination based on specific past medical history, vaccination histories (including dates and times), appropriate for the individual.

State requirements for pre-enrollment vaccination ensure the best protection against widespread measles transmission among students at college campuses and other post-high school educational institutions. Students who do not have documentation of live MMRV vaccination or other acceptable evidence of immunity at the time of enrollment should be admitted to classes only after receiving the first dose of MMRV vaccine. Provide resources and assistance for providers to implement protocols or standing orders, where feasible, and other systems changes to improve routine assessment of vaccine needs and vaccination. Integrate educational information on immunizations into professional training, including training of students in undergraduate and postgraduate training programs should be established.

\section{Conflict of Interest}

No conflict of interest associated with this work.

No commercial relationship or potential conflict of interest related to the submission of this manuscript.

\section{References}

1. CDC (2011) Immunization of Health-Care Personnel. Recommendations of the Advisory Committee on Immunization Practices (ACIP). MMWR 60: 10-25.

2. Numazaki K (2015) Study on assays for the detection of serum antibodies to measles from children and its standardization. Int J Pediatr Neonat Care 1: 108.

3. Davis RM, Orenstein WA, Frank JA Jr, Sacks JJ, Dales LG, et al. (1984) Transmission of measles in medical settings. 1980 through 1984. JAMA 255: 1295-1298. [Crossref]

4. Numazaki K (2007) Current problems of measles control in Japan and western pacific region. Vaccine 25: 3101-3104. [Crossref]

5. Numazaki K, Inoue A, Wang WW (2013) Prevalence of serum antibodies agains vaccine-preventable diseases in students of a Japanese university of health and welfare and its advisory standard of vaccination for clinical training. Eur J Basic Med Sci 3: 50-55.

6. World Health Organization (2009) Global elimination of measles: report by the Secretariat. IRIS.

7. Craig AT, Heywood AE, Worth H (2020) Measles epidemic in Samoa and other Pacific islands. Lancet 20: 273-275. [Crossref]

8. Markowitz LE, Preblud SR, Fine PE, Orenstein WA (1990) Duration of live measles vaccine-induced immunity. Pediatr Infect Dis J 9: 101-110. [Crossref]

9. Hasegawa T, Asaeda A, Hamaguchi Y, Numazaki K (2009) Development of rapid diagnostic reagent for measles. Hybridoma 28: 241-249. [Crossref]

10. Numazaki K (2012) A study on the clinical application of a rapid diagnostic reagent for measles. Eur J Gen Med 9: 39-44.

11. Mathias RG, Meekison WG, Arcand TA, Schechter MA (1989) The role of secondary vaccine failures in measles outbreaks. Am J Public Health 79: 475-478. [Crossref]

12. Edmonson MB, Davis JP, Hopfensperger DJ, Berg JL, Payton LA (1996) Measles vaccination during the respiratory virus season and risk of vaccine failure. Pediatrics 98: 905-10. [Crossref]

13. Ikeda Y, Noda J, Inoue Y, Isogai N (2013) Seroprevalence survey of measles, mumps, rubella and varicella antibodies and measles vaccination in Japanese health care workers. European Journal of Health 1: 1-10.

14. Falk WA, Buchan K, Dow M, Garson JZ, Hill E, et al. (1989) The epidemiology of mumps in Southern Alberta, 1980-1982. Am J Epidemiol 130: 736-749. [Crossref]

15. CDC (1984) Mumps surveillance, January 1977-December 1982. Atlanta, US Department of Health and Human Services, US Public Health Service.

16. Asari S, Deguchi M, Tahara K, Taniike M, Toyokawa M, et al. (2003) Seroprevalence survey of measles, rubella, varicella, and mumps antibodies in health care workers and evaluation of a vaccination program in a tertiary care hospital in Japan. Am J Infect Control 31: 157-162. [Crossref]

17. Grabowsky M, Markowitz L (1991) Serologic screening, mass immunization, and implications for immunization programs (letter). J Infect Dis 164: 1237-1238.

18. National Vaccine Advisory Committee (2014) Recommendations from the National Vaccine Advisory Committee: standards for adult immunization practice. Public Health Rep 129: 115-123. [Crossref]

Copyright: (C2021 Numazaki K. This is an open-access article distributed under the terms of the Creative Commons Attribution License, which permits unrestricted use, distribution, and reproduction in any medium, provided the original author and source are credited. 